\title{
Can local bulk effects explain the galactic dark matter?
}

\author{
Malihe Heydari-Fard ${ }^{1,2 *}$ and Hamid R. Sepangi ${ }^{\dagger}$ \\ ${ }^{1}$ Department of Physics, Shahid Beheshti University, Evin, Tehran 19839, Iran \\ ${ }^{2}$ Department of Physics, The University of Qom, Qom 37185-359, Iran
}

December 21, 2018

\begin{abstract}
We obtain the virial theorem within the context of a brane-world model without mirror symmetry or any form of junction condition. Taking a constant curvature bulk (neglecting non-local bulk effects), the local bulk effects generate a geometrical mass, contributing to the gravitational energy which may be used to explain the virial mass discrepancy in clusters of galaxies. We fix the parameter of this model in agreement with observational data.

PACS numbers: 04.50.-h, 04.20.Jb, 98.35.Ce
\end{abstract}

\section{Introduction}

The question of dark matter is one of the most intriguing aspects of the present-day observational astrophysics and cosmology. Today, its existence at the galactic and extra-galactic scales is well established and is based on two important observations, namely the mass discrepancy in clusters of galaxies and the behavior of the galactic rotation curves [1]. Galaxy clusters are the largest virialized structures in the universe, and their mass content is supposed to be representative of the universe as a whole. The total mass of galaxy clusters ranges from $10^{13} M_{\odot}$ for groups up to a few $10^{15} M_{\odot}$ for very rich systems. Cluster masses can be determined in a variety of ways. The application of the virial theorem to positions and velocities of cluster member galaxies is the oldest method of cluster mass determination [2]. More recent methods are based on the dynamical analysis of hot X-ray emitting gas [3] and on the gravitational lensing of background galaxies [4]. The mass determined from such dynamical means is always found to be in excess of that which can be attributed to the visible matter. This is known as the "missing mass problem." The existence of dark matter was not firmly established until the measurement of the rotational velocity of stars and gases orbiting at a distance $r$ from the galactic center could be done with reasonable accuracy. Observations show that the rotational velocity increases near the center of a galaxy and approaches a nearly constant value with increasing distance from the center. The discrepancy between the observed rotational velocity curves and the theoretical prediction from Newtonian mechanics is known as the "galactic rotation curves problem." This discrepancy is explained by postulating that every galaxy and cluster of galaxies is embedded in a halo made up of some dark (invisible) matter.

The idea that our familiar 4-dimensional $(4 D)$ space-time is a hypersurface (brane) embedded in a $5 \mathrm{D}$ bulk $[5,6,7]$ has been experiencing a phenomenal interest during the last decade. According to this brane-world scenario, all matter and gauge interactions reside on the brane, while gravity can propagate in the whole 5D space-time. Several brane-world cosmologies have been proposed in the context of the Randall-Sundrum (RS) formulations [6], defined in a 5D anti-de Sitter space-time $\left(\mathrm{AdS}_{5}\right)$. The dynamics of these models feature boundary terms in the action and sometimes mirror

*email: m.heydarifard@mail.sbu.ac.ir

${ }^{\dagger}$ email: hr-sepangi@sbu.ac.ir 
symmetry, such that bulk gravitational waves interfere with the brane-world motion. This usually comes together with junction conditions producing an algebraic relationship between the extrinsic curvature and the confined matter $[8,9]$. The consequence is that the Friedman equation acquires an additional term which is proportional to the square of energy density of the confined matter field $[10,11]$. This term was initially considered as a possible solution to the accelerated expansion of the universe. However, soon it was realized to be incompatible with the big bang nucleosynthesis, requiring additional fixes [11].

Brane-world scenarios under more general conditions and still compatible with the brane-world program have also been rather extensively studied over the past decade where it has been shown that it is possible to find a richer set of cosmological solutions in accordance with the current observations [12]. Under these conditions, without using $Z_{2}$ symmetry or without postulating any junction condition, Friedman equation is modified by a geometrical term which is defined in terms of the extrinsic curvature, leading to a geometrical interpretation for dark energy [13]. There has also been arguments concerning the uniqueness of the junction conditions. Indeed, other forms of junction conditions exist, so that different conditions may lead to different physical results [9]. Furthermore, these conditions cannot be used when more than one non-compact extra dimension is involved. Against this background, an interesting higher-dimensional model was introduced in [14] where particles are trapped on a 4D hypersurface by the action of a confining potential. The dynamics of test particles confined to a brane by the action of such potential at the classical and quantum levels were studied in [15]. In [16], the same brane-world model was studied, offering a geometrical explanation for the accelerated expansion of the universe. A geometrical explanation for the generalized Chaplygin gas was considered in [17] along the same lines. We have also studied exact solutions of the vacuum field equations on the brane for two interesting cases. The first solution can be used to explain the galaxy rotation curves without assuming the existence of dark matter and without having to resort to the Modified Newtonian Dynamics (MOND) $[18,19]$, and the second solution represents a black hole in an asymptotically de Sitter space-time [20].

It has been argued that modified theories of gravity based on the RS brane-world scenario can explain the observed galactic rotation curves without introducing any additional hypothesis. In such models, a spherically symmetric brane-world metric results in a Schwarzschild mass parameter receiving a new contribution due to the Weyl tensor which is interpreted as the mass of dark matter [21]. Neglecting the cosmological constant, the dark mass increases linearly with the radial distance from the galactic center, explaining the flatness of the galactic rotation curves. The bending angle of light predicted by brane-world modeles was found to be much larger compared to the predictions of dark matter models, the deviation increasing with the degree of compactness [22]. Using the smallness of the rotational velocity, a perturbation scheme for reconstructing the metric in a galactic halo in brane-world models with induced gravity was developed in [23]. It has also been shown that the non-standard model bulk fields can replace dark matter in explaining structure formation, as the evolution on the brane becomes similar to that of the cold dark matter [24]. Similar interpretations of dark matter as bulk effects have also been considered in $[25,26]$.

As was mentioned above, the galactic dynamics of massive test particles may be understood without resorting to dark matter in the framework of $f(R)$ modified theories of gravity [27]. Recently, it has been argued that the virial theorem mass discrepancy in clusters of galaxies can be accounted for within the context of the RS model and $f(R)$ modified theories of gravity [28, 29]. It would therefore be of interest to study the latter in a brane-world model with a constant curvature bulk without using the $Z_{2}$ symmetry or without postulating any form of junction conditions $[12,13]$. In so doing the gravitational field equations on the brane are modified by a local extra term, $Q_{\mu \nu}$. Using the collisionless Boltzmann equation, we obtain the virial theorem which is modified by an extra term that may be used to explain the virial mass discrepancy in clusters of galaxies. 


\section{Field equations for the brane-world}

The embedding of the brane-world in the bulk plays an essential role in the covariant formulation of the brane-world gravity, since it tells us how the Einstein-Hilbert dynamics of the bulk is transferred to the brane-world. However, there are many different ways to embed a manifold into another, classified as local, global, isometric, conformal, rigid, deformable, analytic or differentiable. The choice of one or other depends on what the embedded manifold is supposed to do.

Generally, there are three basic postulates in the geometrical approach considered in brane-world scenarios, that is, the confinement of the standard gauge interactions to the brane, the existence of quantum gravity in the bulk and finally, the embedding of the brane-world. All other model dependent properties such as warped metric, mirror symmetries, radion or extra scalar fields, fine tuning parameters like the tension of the brane and the choice of a junction condition are left out as much as possible in our calculations [12].

In the following we extend this embedding to any constant curvature space, including anti-de Sitter $A d S_{5}$, de Sitter $d S_{5}$, and the flat $M_{5}$ cases, with the bulk Riemann tensor written as

$$
\mathcal{R}_{A B C D}=k_{*}\left(\mathcal{G}_{A C} \mathcal{G}_{B D}-\mathcal{G}_{A D} \mathcal{G}_{B C}\right),
$$

where $\mathcal{G}_{A B}$ is the bulk metric. In the normal Gaussian frame defined by the embedded space-time, it may be decomposed as

$$
\mathcal{G}_{A B}=\left(\begin{array}{cc}
g_{\mu \nu} & 0 \\
0 & g_{55}
\end{array}\right), \quad g_{55}=\varepsilon= \pm 1
$$

where $k_{*}$ denotes the bulk constant curvature. In the flat case $k_{*}=0$ and we may write $k_{*}=\varepsilon \frac{\Lambda^{(b)}}{6}$, where $\Lambda^{(b)}$ is the bulk cosmological constant. We note that for a $d S_{5}$ bulk $\varepsilon=+1$ and for a $A d S_{5}$ bulk $\varepsilon=-1$. With this assumption the Gauss-Codazzi equations [30]

$$
\begin{gathered}
R_{\alpha \beta \gamma \delta}=\frac{1}{\varepsilon}\left(K_{\alpha \gamma} K_{\beta \delta}-K_{\alpha \delta} K_{\beta \gamma}\right)+\mathcal{R}_{A B C D} \mathcal{Z}_{, \alpha}^{A} \mathcal{Z}_{, \beta}^{B} \mathcal{Z}_{, \gamma}^{C} \mathcal{Z}_{, \delta}^{D}, \\
K_{\alpha[\beta ; \gamma]}=\mathcal{R}_{A B C D} \mathcal{Z}_{, \alpha}^{A} \mathcal{N}^{B} \mathcal{Z}_{, \beta}^{C} \mathcal{Z}_{, \gamma}^{D}
\end{gathered}
$$

which are the integrability conditions for the embedding equations, reduce to

$$
\begin{gathered}
R_{\alpha \beta \gamma \delta}=\frac{1}{\varepsilon}\left(K_{\alpha \gamma} K_{\beta \delta}-K_{\alpha \delta} K_{\beta \gamma}\right)+k_{*}\left(g_{\alpha \gamma} g_{\beta \delta}-g_{\alpha \delta} g_{\beta \gamma}\right), \\
K_{\alpha[\beta ; \gamma]}=0
\end{gathered}
$$

where $K_{\mu \nu}$ denotes the extrinsic curvature. From the contractions of equation (5) we can obtain the Ricci scalar $R$ of the brane metric, in terms of the Ricci scalar of the bulk $\mathcal{R}$. After adding a confined matter Lagrangian and a four dimensional cosmological constant $\Lambda$, the effective Lagrangian compatible with the embedding reads, for details see [31]

$$
\mathcal{L}_{e f f}=R \sqrt{-g}-\frac{1}{\varepsilon}\left(K^{\mu \nu} K_{\mu \nu}+K^{2}\right) \sqrt{-g}+\mathcal{R} \sqrt{-g}-\frac{2}{\varepsilon} \frac{d K}{d y} \sqrt{-g}+\Lambda \sqrt{-g}-\mathcal{L}_{m}
$$

where $K=g^{\mu \nu} K_{\mu \nu}$ is the mean curvature of the brane-world, $y$ denotes the fifth coordinate and $\mathcal{R}=-20 k_{*}$ as derived from equation (1). The total derivative term with respect to $y$ can be eliminated provided the motion of the brane-world occurs between two fixed minimal boundary hypersurfaces where $K=0$ [12]. Similar to the RSII model, such boundaries can be moved away so that all 
boundary generated bulk waves are eliminated. Variation of the action with respect to $g_{\mu \nu}$ gives the dynamical equation compatible with the embedding and with the confined matter hypotheses

$$
G_{\mu \nu}=8 \pi G \tau_{\mu \nu}-\lambda g_{\mu \nu}+Q_{\mu \nu}
$$

where

$$
Q_{\mu \nu}=\frac{1}{\varepsilon}\left[K_{\mu}^{\rho} K_{\rho \nu}-K K_{\mu \nu}-\frac{1}{2}\left(K_{\alpha \beta} K^{\alpha \beta}-K^{2}\right) g_{\mu \nu}\right] .
$$

Here, $\tau_{\mu \nu}$ is the confined matter energy-momentum tensor on the brane and $\lambda=-3 k_{*}+\Lambda$. As can be seen from definition of $Q_{\mu \nu}$, it is independently a conserved quantity, that is

$$
Q_{; \mu}^{\mu \nu}=0
$$

so that there is no non-gravitational exchange of energy between this geometrical correction and the confined matter. Such an aspect has one important consequence, that is, if $Q_{\mu \nu}$ is to be related to dark energy, it does not exchange energy with ordinary matter, much the same as in coupled quintessence models [32].

In order to solve the Codazzi equation (6), we choose the static spherically symmetric metric on the brane in the form

$$
d s^{2}=-e^{\mu(r)} d t^{2}+e^{\nu(r)} d r^{2}+r^{2}\left(d \theta^{2}+\sin ^{2} \theta d \varphi^{2}\right) .
$$

Then the York relation

$$
K_{\mu \nu}=-\frac{1}{2} \frac{\partial g_{\mu \nu}}{\partial \xi}
$$

shows that in a diagonal metric, $K_{\mu \nu}$ are diagonal. Here, $\xi$ is a small parameter orthogonal to the brane that parameterizes the extra noncompact dimension. Now, separating the spatial components, the Codazzi equation reduces to

$$
\begin{gathered}
K_{\mu \nu, \sigma}-K_{\nu \rho} \Gamma_{\mu \sigma}^{\rho}=K_{\mu \sigma, \nu}-K_{\sigma \rho} \Gamma_{\mu \nu}^{\rho} \\
K_{00,1}-\left(\frac{\mu^{\prime}}{2}\right) K_{00}=-\left(\frac{\mu^{\prime} e^{\mu}}{2 e^{\nu}}\right) K_{11} \\
K_{22,1}-\left(\frac{1}{r}\right) K_{22}=\left(r e^{-\nu}\right) K_{11} .
\end{gathered}
$$

The first equation gives $K_{00, \sigma}=K_{11, \sigma}=K_{22, \sigma}=K_{33, \sigma}=0$ for $\sigma=0,3$. Repeating the same procedure for $\sigma=2$, we obtain $K_{00, \sigma}=K_{11, \sigma}=K_{22, \sigma}=0$. This shows that $K_{11}$ depends only on the variable $r$. Assuming $K_{11}=\alpha e^{\nu(r)}$ and using equations (14) and (15), one finds

$$
\begin{gathered}
K_{00}(r)=-\alpha e^{\mu(r)}+c e^{\mu(r) / 2}, \\
K_{22}(r)=\alpha r^{2}+\beta r .
\end{gathered}
$$

Taking $\mu, \nu=3$ in the first equation we obtain

$$
\begin{gathered}
K_{33,1}-\left(\frac{1}{r}\right) K_{33}=\left(e^{-\nu} r \sin ^{2} \theta\right) K_{11}=\alpha r \sin ^{2} \theta \\
K_{33,2}-(\cot \theta) K_{33}=(\sin \theta \cos \theta) K_{22} .
\end{gathered}
$$


Using equations (16), (17) and (18) then results in

$$
K_{33}(r, \theta)=\alpha r^{2} \sin ^{2} \theta+r \beta \sin ^{2} \theta+r c_{1} \sin \theta .
$$

Now, use of equation (9) leads to the components of $Q_{\mu \nu}$

$$
\begin{aligned}
Q_{00} & =-\frac{g_{00}}{\varepsilon r^{2}}\left[3 \alpha^{2} r^{2}+4 \alpha \beta r+\beta^{2}+\frac{c_{1}}{\sin \theta}(2 \alpha r+\beta)\right] \\
Q_{11} & =-\frac{g_{11}}{\varepsilon r^{2}}\left[3 \alpha^{2} r^{2}+4 \alpha \beta r+\beta^{2}+\frac{c_{1}}{\sin \theta}\left(2 \alpha r+\beta-c r e^{-\mu / 2}\right)-2 c e^{-\mu / 2}\left(\alpha r^{2}+\beta r\right)\right], \\
Q_{22} & =\frac{g_{22}}{\varepsilon r}\left[-3 \alpha^{2} r-2 \alpha \beta+c e^{-\mu / 2}(2 \alpha r+\beta)+\frac{c_{1}}{\sin \theta}\left(-2 \alpha+c e^{-\mu / 2}\right)\right], \\
Q_{33} & =\frac{g_{33}}{\varepsilon r}\left[-3 \alpha^{2} r-2 \alpha \beta+c e^{-\mu / 2}(2 \alpha r+\beta)\right] .
\end{aligned}
$$

Since $G_{2}^{2}=G_{3}^{3}$ and thus $Q_{2}^{2}=Q_{3}^{3}$, one obtains $c_{1}=0$. Now, using these relations and equation (8), the gravitational field equations become

$$
\begin{gathered}
e^{-\nu}\left(-\frac{1}{r^{2}}+\frac{\nu^{\prime}}{r}\right)+\frac{1}{r^{2}}=8 \pi G \rho_{e f f}+\lambda+\frac{1}{\varepsilon}\left(3 \alpha^{2}+\frac{4 \alpha \beta}{r}+\frac{\beta^{2}}{r^{2}}\right) \\
e^{-\nu}\left(\frac{1}{r^{2}}+\frac{\mu^{\prime}}{r}\right)-\frac{1}{r^{2}}=8 \pi G p_{e f f}^{(r)}-\lambda+\frac{1}{\varepsilon}\left(-3 \alpha^{2}+2 \alpha c e^{-\mu / 2}-\frac{4 \alpha \beta}{r}-\frac{\beta^{2}}{r^{2}}+\frac{2 \beta c}{r} e^{-\mu / 2}\right), \\
e^{-\nu}\left(\frac{\mu^{\prime}}{r}-\frac{\nu^{\prime}}{r}-\frac{\mu^{\prime} \nu^{\prime}}{2}+\mu^{\prime \prime}+\frac{\mu^{\prime 2}}{2}\right)=16 \pi G p_{e f f}^{(\perp)}-2 \lambda+\frac{1}{\varepsilon}\left(-6 \alpha^{2}+4 \alpha c e^{-\mu / 2}-\frac{4 \alpha \beta}{r}+\frac{2 \beta c}{r} e^{-\mu / 2}\right),
\end{gathered}
$$

where a prime represents differentiation with respect to $r$ and $\varepsilon$ is the signatures of the bulk space, $\varepsilon=+1$ for the $d S_{5}$ bulk and $\varepsilon=-1$ for the $A d S_{5}$ bulk. In the above equations we have assumed that the matter on the brane consists of an anisotropic fluid, characterized by an effective energy density $\rho_{\text {eff }} \neq 0$, a radial pressure $p_{\text {eff }}^{(r)}$ and a tangential pressure $p_{\text {eff }}^{(\perp)}$.

In the next section, we will investigate the influence of the extra term, $Q_{\mu \nu}$, on the dynamics of the galaxies by adopting the method introduced in [28].

\section{Dark matter and the role of extrinsic curvature}

In order to obtain the virial theorem for galaxy clusters in our model, we have to write down the general relativistic Boltzmann equation governing the evolution of the distribution function $f$. Galaxies, which are treated as identical and collisionless point particles, are described by this distribution function. For the static spherically symmetric metric given by equation (11) we introduce the following frame of orthonormal vectors $[33,34]$

$$
e_{\rho}^{(0)}=e^{\mu / 2} \delta_{\rho}^{0}, \quad e_{\rho}^{(1)}=e^{\nu / 2} \delta_{\rho}^{1}, \quad e_{\rho}^{(2)}=r \delta_{\rho}^{2}, \quad e_{\rho}^{(3)}=r \sin \theta \delta_{\rho}^{3},
$$

where $g^{\mu \nu} e_{\mu}^{(a)} e_{\nu}^{(b)}=\eta^{(a)(b)}$. The four velocity $v^{\mu}$ of a typical galaxy, satisfying the condition $v^{\mu} v_{\mu}=$ -1 , in tetrad components is given by

$$
v^{(a)}=v^{\mu} e_{\mu}^{(a)} .
$$

The relativistic Boltzmann equation in tetrad components is given by

$$
v^{(a)} e_{(a)}^{\rho} \frac{\partial f}{\partial x^{\rho}}+\gamma_{(b)(c)}^{(a)} v^{(b)} v^{(c)} \frac{\partial f}{\partial v^{(a)}}=0
$$


where the distribution function $f=f\left(x^{\mu}, v^{(a)}\right), a=0,1,2,3$, and $\gamma_{(b)(c)}^{(a)}=e_{\rho ; \sigma}^{(a)} e_{(b)}^{\rho} e_{(c)}^{\sigma}$ are the Ricci rotation coefficients. Thus, equation (27) becomes

$$
\begin{aligned}
v_{r} \frac{\partial f}{\partial r} & +\frac{e^{\nu / 2} v_{\theta}}{r} \frac{\partial f}{\partial \theta}+\frac{e^{\nu / 2} v_{\varphi}}{r \sin \theta} \frac{\partial f}{\partial \varphi}-\left(\frac{v_{t}^{2}}{2} \frac{\partial \mu}{\partial r}-\frac{v_{\theta}^{2}+v_{\varphi}^{2}}{r}\right) \frac{\partial f}{\partial v_{r}} \\
& -\frac{v_{r}}{r}\left(v_{\theta} \frac{\partial f}{\partial v_{\theta}}+v_{\varphi} \frac{\partial f}{\partial v_{\varphi}}\right)-\frac{e^{\nu / 2} v_{\varphi}}{r} \cot \theta\left(v_{\theta} \frac{\partial f}{\partial v_{\varphi}}-v_{\varphi} \frac{\partial f}{\partial v_{\theta}}\right)=0 .
\end{aligned}
$$

Assuming that the distribution function is only a function of the radial coordinate $r$, equation (28) reduces to

$$
v_{r} \frac{\partial f}{\partial r}-\left(\frac{v_{t}^{2}}{2} \frac{\partial \mu}{\partial r}-\frac{v_{\theta}^{2}+v_{\varphi}^{2}}{r}\right) \frac{\partial f}{\partial v_{r}}-\frac{v_{r}}{r}\left(v_{\theta} \frac{\partial f}{\partial v_{\theta}}+v_{\varphi} \frac{\partial f}{\partial v_{\varphi}}\right)-\frac{e^{\nu / 2} v_{\varphi}}{r} \cot \theta\left(v_{\theta} \frac{\partial f}{\partial v_{\varphi}}-v_{\varphi} \frac{\partial f}{\partial v_{\theta}}\right)=0
$$

Now, taking into account the spherically symmetric nature of the problem at hand and integrating over the velocity space and assuming that the distribution function vanishes rapidly as the velocities tend to $\pm \infty$, one obtains

$$
r \frac{\partial}{\partial r}\left[\rho\left\langle v_{r}^{2}\right\rangle\right]+\frac{1}{2} \rho\left[\left\langle v_{t}^{2}\right\rangle+\left\langle v_{r}^{2}\right\rangle\right] r \frac{\partial \mu}{\partial r}-\rho\left[\left\langle v_{\theta}^{2}\right\rangle+\left\langle v_{\varphi}^{2}\right\rangle-2\left\langle v_{r}^{2}\right\rangle\right]=0 .
$$

Multiplying equation (30) by $4 \pi r^{2}$ and integrating over the cluster leads to

$$
-\int_{0}^{R} 4 \pi \rho\left[\left\langle v_{r}^{2}\right\rangle+\left\langle v_{\theta}^{2}\right\rangle+\left\langle v_{\varphi}^{2}\right\rangle\right] r^{2} d r+\frac{1}{2} \int_{0}^{R} 4 \pi r^{3} \rho\left[\left\langle v_{t}^{2}\right\rangle+\left\langle v_{r}^{2}\right\rangle\right] \frac{\partial \mu}{\partial r} d r=0 .
$$

Now, using the total kinetic energy of the galaxies

$$
k=\int_{0}^{R} 2 \pi \rho\left[\left\langle v_{r}^{2}\right\rangle+\left\langle v_{\theta}^{2}\right\rangle+\left\langle v_{\varphi}^{2}\right\rangle\right] r^{2} d r
$$

equation (31) is reduced to

$$
2 k-\frac{1}{2} \int_{0}^{R} 4 \pi r^{3} \rho\left[\left\langle v_{t}^{2}\right\rangle+\left\langle v_{r}^{2}\right\rangle\right] \frac{\partial \mu}{\partial r} d r=0 .
$$

Let us write the energy-momentum tensor of the confined matter on the brane in terms of the distribution function

$$
\tau_{\mu \nu}=\int f m v_{\mu} v_{\nu} d v
$$

which gives

$$
\rho_{\text {eff }}=\rho\left\langle v_{t}^{2}\right\rangle, \quad p_{\text {eff }}^{(r)}=\rho\left\langle v_{r}^{2}\right\rangle, \quad p_{\text {eff }}^{(\perp)}=\rho\left\langle v_{\theta}^{2}\right\rangle=\rho\left\langle v_{\varphi}^{2}\right\rangle .
$$

Now, using these relations and summing the gravitational field equations (22)-(24) we find

$$
e^{-\nu}\left(\frac{\mu^{\prime}}{r}-\frac{\mu^{\prime} \nu^{\prime}}{4}+\frac{\mu^{\prime \prime}}{2}+\frac{\mu^{\prime 2}}{4}\right)=4 \pi G \rho\left\langle v^{2}\right\rangle-\lambda+\frac{1}{\varepsilon}\left(-3 \alpha^{2}-\frac{2 \alpha \beta}{r}+3 \alpha c e^{-\mu / 2}+\frac{2 \beta c}{r} e^{-\mu / 2}\right),
$$

where $\left\langle v^{2}\right\rangle=\left\langle v_{t}^{2}\right\rangle+\left\langle v_{r}^{2}\right\rangle+\left\langle v_{\theta}^{2}\right\rangle+\left\langle v_{\varphi}^{2}\right\rangle$. In order to obtain the generalized virial theorem in our model we have to use some approximations and assumptions. Since the dispersion of the velocity of galaxies in the clusters is of the order $600-1000 \mathrm{~km} / \mathrm{s}$, that is $\left(\frac{v}{c}\right)^{2} \approx 4 \times 10^{-6}-1.11 \times 10^{-5} \ll 1$, therefore we can neglect the relativistic effects in the Boltzmann equation, and use the small velocity limit approximation, so that $\left\langle v_{r}^{2}\right\rangle \approx\left\langle v_{\theta}^{2}\right\rangle \approx\left\langle v_{\varphi}^{2}\right\rangle \ll\left\langle v_{t}^{2}\right\rangle \approx 1$. The intensity of the gravitational effects can be estimated from the ratio of the Schwarzschild radius to the radius of cluster, $G M / R$, which for 
typical clusters is of the order of $10^{-6} \ll 1$. Therefore the gravitational field inside galactic clusters is weak and we can use the weak gravitational field approximation, so that the quadratic terms can be neglected [28]. Thus, equation (36) is given by

$$
\frac{1}{2 r^{2}} \frac{\partial}{\partial r}\left(r^{2} \frac{\partial \mu}{\partial r}\right)=4 \pi G \rho-\lambda+4 \pi G \rho_{e x t r}
$$

where

$$
4 \pi G \rho_{\text {extr }}=\frac{1}{\varepsilon}\left(-3 \alpha^{2}-\frac{2 \alpha \beta}{r}+3 \alpha c e^{-\mu / 2}+\frac{2 \beta c}{r} e^{-\mu / 2}\right),
$$

and the suffix 'extr' stands for 'extrinsic'. Multiplying equation (37) by $r^{2}$ and integrating from 0 to $r$ we obtain

$$
\frac{1}{2} r^{2} \frac{\partial \mu}{\partial r}-G M(r)+\frac{1}{3} \lambda r^{3}-4 \pi G \int_{0}^{r} \rho_{e x t r}\left(r^{\prime}\right) r^{\prime 2} d r^{\prime}=0
$$

where $M(r)$ is the mass out to radius $r$, so that $d M(r)=4 \pi \rho r^{2} d r$. The total baryonic mass of the system is given by $M=\int_{0}^{r} 4 \pi \rho r^{\prime 2} d r^{\prime}$. We also define the geometrical mass as

$$
M_{\text {extr }}(r)=4 \pi \int_{0}^{r} \rho_{\text {extr }}\left(r^{\prime}\right) r^{\prime 2} d r^{\prime}
$$

Multiplying equation (39) by $d M(r)$ and integrating from 0 to $R$ and introducing the moment of inertia of the system as $I=\int_{0}^{R} r^{2} d M(r)$, we obtain

$$
2 k+W+\frac{1}{3} \lambda I-W_{\text {extr }}=0 .
$$

In the above equation we have used equation (32) and the following definitions

$$
W=-\int_{0}^{R} \frac{G M(r)}{r} d M(r),
$$

and

$$
W_{\text {extr }}=\int_{0}^{R} \frac{G M_{\text {extr }}(r)}{r} d M(r),
$$

where $W$ is the gravitational potential energy of the system. For the case $\alpha=\beta=c=0$, we obtain $W_{\text {extr }}=0$ and equation (41) reduces to the virial theorem in the presence of a cosmological constant [33]. Now, let us introduce the radii $R_{v}, R_{I}$ and $R_{\text {extr }}$ as [28]

$$
\begin{gathered}
R_{v}=\frac{M^{2}}{\int_{0}^{R} \frac{M(r) d M(r)}{r}}, \\
R_{I}^{2}=\frac{\int_{0}^{R} r^{2} d M(r)}{M(r)}, \\
R_{\text {extr }}=\frac{M_{\text {extr }}^{2}}{\int_{0}^{R} \frac{M_{\text {extr }}(r) d M(r)}{r}},
\end{gathered}
$$

where $R_{v}$ is the virial radius and $R_{e x t r}$ is defined as the geometrical radius of the clusters of galaxies. Defining the virial mass as [33]

$$
2 k=\frac{G M_{v}^{2}}{R_{v}}
$$


and using the following relations

$$
W=-\frac{G M^{2}}{R_{v}}, \quad W_{e x t r}=\frac{G M_{e x t r}^{2}}{R_{e x t r}}, \quad I=M R_{I}^{2},
$$

the generalized virial theorem (41) is simplified as

$$
\frac{M_{v}}{M}=\left(1-\frac{\lambda R_{v} R_{I}^{2}}{3 G M}+\frac{M_{e x t r}^{2} R_{v}}{M^{2} R_{e x t r}}\right)^{1 / 2}
$$

As can be seen, we have three type of mass in equation (49), namely, the total baryonic mass of the system represented by $M$ (including the baryonic mass of the intra-cluster gas and of the stars, other particles like massive neutrinos), the virial mass represented by $M_{v}$ and finally, the geometrical mass represented by $M_{\text {extr }}$.

On large distance scales associated with galaxies, we can ignore the contribution of the cosmological constant relative to the mass energy of the galaxy. Since, as it turns out, $M_{v}$ is considerably greater than $M$ for most of the clusters, we can neglect the unitary term in equation (49). Therefore, the virial mass is given by

$$
M_{v} \approx M_{e x t r}\left(\frac{R_{v}}{R_{e x t r}}\right)^{1 / 2} .
$$

This equation shows that the virial mass is proportional to the geometrical mass due to the local extra term, $Q_{\mu \nu}$, thus the virial mass discrepancy in clusters of galaxies can be explained by the generalized virial theorem. Since galactic clusters are dark matter dominated objects, the main contribution to their mass comes from the geometrical mass $M_{\text {extr }}$ so that with a very good approximation $M_{\text {extr }} \approx$ $M_{\text {total }}$.

All the way, among various possible solutions that depend on different choices of arbitrary constants $\alpha, \beta, c$, we consider $\alpha=0$ and $\beta, c \neq 0$. Since the bulk of matter in clusters of galaxies is in the form of dark matter, we can neglect the effect of the ordinary matter in equations (22)-(24). Thus, the solutions are given by

$$
\begin{aligned}
& e^{-\nu}=\left(1-\frac{\beta^{2}}{\varepsilon}\right), \\
& e^{-\mu / 2}=\frac{\left(\varepsilon-\beta^{2}\right)}{\beta c} \frac{1}{r} .
\end{aligned}
$$

Now, using equations (38) and (40) the geometrical mass is given by

$$
G M_{e x t r}(r)=\frac{1}{\varepsilon} \int_{0}^{r}\left[-3 \alpha^{2}-\frac{2 \alpha \beta}{r^{\prime}}+3 \alpha c e^{-\mu\left(r^{\prime}\right) / 2}+\frac{2 \beta c}{r^{\prime}} e^{-\mu\left(r^{\prime}\right) / 2}\right] r^{\prime 2} d r^{\prime} .
$$

Substituting $e^{-\mu / 2}$ from equation (52), we obtain

$$
G M_{e x t r}(r)=2\left(1-\frac{\beta^{2}}{\varepsilon}\right) r .
$$

Since according to our physical interpretation $M_{\text {extr }}$ is an effective geometrical mass it must satisfy the condition $M_{\text {extr }}>0$, therefore we take $\varepsilon=-1$ ( $A d S_{5}$ bulk). This geometrical mass is linearly increasing with $r$, thus having a similar behavior as that of dark matter in clusters of galaxies. An important observational quantity is the radial velocity dispersion $\sigma_{r}^{2}$, which is related to the total mass in a cluster by the relation $G M_{v}\left(R_{v}\right)=\sigma_{r}^{2} R_{v}$. For $r=R_{v}$ equation (54) becomes $G M_{\text {extr }}\left(R_{v}\right) \approx\left(1+\beta^{2}\right) R_{v}$ and assuming that $M_{\text {extr }} \approx M_{v}$ we obtain

$$
\left(1+\beta^{2}\right) \approx \sigma_{r}^{2}
$$


Therefore, the present model gives a geometrical interpretation for the mass discrepancy in clusters of galaxies, supporting our previous results that the galaxy rotation curves can be explained without assuming the existence of dark matter and new modified theories (Modified Newtonian Dynamics) $[20]$.

Finally, we should mention here that there is a difference between our model and the work presented in [28]. In this paper we have shown that the virial mass of galaxy clusters is mainly determined by the geometrical mass associated with the extrinsic curvature terms (local bulk effects) whereas in [28], the virial mass is specified by the dark mass associated with the transmitted projection of the bulk Weyl tensor (non-local bulk effects).

\section{Conclusions}

In the RS brane-world models, the 4-dimensional effective Einstein equation has some extra terms known as dark radiation and dark pressure, which describe the non-local bulk effects due to the gravitational field of the bulk. It has been shown that the dark radiation term can be used to explain the virial theorem mass discrepancy in clusters of galaxies [28].

In the present paper we have generalized the virial theorem within the context of brane-world models without using the $Z_{2}$ symmetry or without postulating any junction condition. To obtain the virial theorem we have used a method based on the collisionless Boltzmann equation. Assuming a constant curvature bulk, the virial theorem was modified by a local extra term, equation (41), which was then used to explain the virial mass discrepancy in clusters of galaxies. Thus, the geometrical mass can play the role of dark matter in clusters of galaxies, supporting our previous results that the rotational galactic curves can be explained in our model without introducing any additional hypothesis [20]. Finally, after fixing the parameter of this model, we found them to be in good agreement with observations.

\section{References}

[1] J. Binney and S. Tremaine, Galactic dynamics, Princeton, Princeton University Press, (1987).

[2] F. Zwicky, Helv. Phys. Acta. 6 (1933) 110.

[3] L. L. Cowie, M. Henriksen and R. Mushotzky Astrophys. J. 317 (1987) 593.

[4] S. A. Grossman and R. Narayan, Astrophys. J. 344 (1989) 637.

[5] N. Arkani-Hamed, S. Dimopoulos, and G. Dvali, Phys. Lett. B 429 (1998) 263;

I. Antoniadis, N. Arkani-Hamed, S. Dimopoulos, and G. Dvali, Phys. Lett. B 436 (1998) 257.

[6] L. Randall and R. Sundrum, Phys. Rev. Lett. 83 (1999) 3370;

L. Randall and R. Sundrum, Phys. Rev. Lett. 83 (1999) 4690.

[7] G. Dvali, G. Gabadadze, and M. Porrati, Phys. Lett. B 485 (2000) 208;

G. Dvali and G. Gabadadze, Phys. Rev. D 63 (2001) 065007.

[8] W. Israel, Nuovo Cimento B 44 (1966) 1.

[9] R. A. Battye and B. Carter, Phys. Lett. B 509 (2001) 331.

[10] J. M. Cline, C. Grojean and G. Servant, Phys. Rev. Lett. 83 (1999) 4245;

T. Shiromizu, K. Maeda and M. Sasaki, Phys. Rev. D 62 (2000) 024012.

[11] P. Binetruy, C. Deffayet and D. Langlois, Nucl. Phys. B 565 (2000) 269;

P. Binetruy, C. Deffayet, U. Ellwanger and D. Langlois, Phys. Lett. B 477 (2000) 285. 
[12] M. D. Maia, E. M. Monte and J. M. F. Maia, Phys. Lett. B 585 (2004) 11.

[13] M. D. Maia, E. M. Monte, J. M. F. Maia and J. S. Alcaniz, Class. Quant. Grav. 22 (2005) 1623.

[14] V. A. Rubakov and M. E. Shaposhnikov, Phys. Lett. B 125 (1983) 136.

[15] S. Jalazadeh and H. R. Sepangi, Class. Quant. Grav. 22 (2005) 2035.

[16] M. Heydari-Fard, M. Shirazi, S. Jalalzadeh and H. R. Sepangi, Phys. Lett. B 640 (2006) 1.

[17] M. Heydari-Fard and H. R. Sepangi, Phys. Rev. D 76 (2007) 104009.

[18] J. Bekenstein and M. Milgrom Astrophys. J 286 (1984) 7,

M. Milgrom New Astron. Rev 46 (2002) 741,

M. Milgrom Astrophys. J 270 (1983) 365, ibid Astrophys. J 599 (2003) L25.

[19] J. W. Moffat and I. Y. Sokolov, Phys. Lett. B 378 (1996) 59,

P. D. Mannheim, Astrophys. J 419 (1993) 150, ibid Astrophys. J 479 (1997) 659.

[20] M. Heydari-Fard, H. Razmi and H. R. Sepangi, Phys. Rev. D 76 (2007) 066002.

[21] M. K. Mak and T. Harko, Phys. Rev. D 70 (2004) 024010;

M. K. Mak and T. Harko, Annals of Physics 319 (2005) 471;

C. G. Boehmer and T. Harko, Class. Quant. Grav. 24 (2007) 3191.

[22] T. Harko and K. S. Cheng, Astrophys. J. 636 (2006) 8.

[23] A. Viznyuk and Y. Shtanov, Phys. Rev. D 76 (2007) 064009.

[24] S. Pal, Phys. Rev. D 74 (2006) 024005.

[25] C. G. Boehmer and T. Harko, Mon. Not. Roy. Astron. Soc. 379 (2007) 393;

C. G. Boehmer and T. Harko, JCAP 06 (2007) 025.

[26] S. Pal, S. Bharadwaj and S. Kar, Phys. Lett. B 609 (2005) 194;

S. Pal, Phys. Teacher 47 (2005) 144.

[27] C. F. Martins and P. Salucci, Mon. Not. Roy. Astron. Soc. 375 (2007);

A. Borowiec, W. Godlowski and M. Szydlowski, Int. J. Geom. Meth. Mod. Phys. 4 (2007) 183;

O. Bertolami, C. G. Boehmer, T. Harko and F. S. N. Lobo, Phys. Rev. D 75 (2007) 104016;

O. Bertolami, C. G. Boehmer, T. Harko and F. S. N. Lobo, Phys. Rev. D 75 (2007) 104016;

S. Capozziello, V. F. Cardone and A. Troisi, JCAP 0608 (2006) 001; ibid Mon. Not. Roy. Astron. Soc. 375 (2007) 1423.

[28] T. Harko and K. S. Cheng, Phys. Rev. D 76 (2007) 044013.

[29] C. G. Boehmer, T. Harko and F. S. N. Lobo, JCAP 03 (2008) 024.

[30] L. P. Eisenhart 1966 Riemannian Geometry, Princeton University Press, Princeton NJ (1966).

[31] M. D. Maia and E. M. Monte, Phys. Lett. A 297 (2002) 9.

[32] D. Tocchini-Valentini and L. Amendola, Phys.Rev. D 65 (2002) 063508;

J. M. F. Maia and J. A. S. Lima, Phys.Rev. D 65 (2002) 083513;

W. Zimdahl, D. Pavon and L. P. Chimento, Phys. Lett. B 521 (2001) 133.

[33] J. C. Jackson, Mon. Not. Roy. Astron. Soc. 148 (1970) 249. 
[34] R. W. Lindquist, Annals of Physics 37 (1966) 487;

R. Maartens and S. D. Maharaj, J. Math. Phys. 26 (1985) 2869;

S. Bildhauer, Class. Quant. Grav. 6 (1989) 1171;

Z. Banach and S. Piekarski, J. Math. Phys. 35 (1994) 4809. 